\title{
Existence and Uniqueness of Entropy Solution for an Elliptic Problem with Nonlinear Boundary Conditions and Measure-data
}

\author{
Arouna Ouedraogo \\ Department of Mathematics, Norbert Zongo University, Koudougou, Burkina Faso
}

Email address:

arounaoued2002@yahoo.fr, arouna.ouedraogo@unz.bf

\section{To cite this article:}

Arouna Ouedraogo. Existence and Uniqueness of Entropy Solution for an Elliptic Problem with Nonlinear Boundary Conditions and Measure-data. American Journal of Applied Mathematics. Vol. 7, No. 4, 2019, pp. 114-126. doi: 10.11648/j.ajam.20190704.13

Received: July 15, 2019; Accepted: August 29, 2019; Published: September 16, 2019

\begin{abstract}
In this paper, we study a class of elliptic equations on a bounded domain with nonlinear boundary conditions of type graph and measure - data. First of all, give some spaces and basic assumptions. Next, we apply the classical variational approach. So, we need an essentially bounded estimate on the solution, which is not evident to obtain directly in our problem. The obstacles which we encounter is that we cannot get rid of the non-linear term evaluated as a zero gradient and it appear at the boundary, for the part of the measure-data, a term which cannot vanish, when one uses the integration by parts formula. To overcome this difficulties, we first redefine and extend the function which appears in the third Leray-Lions-type conditions and we add a penalization term on the boundary. Secondly, we consider a smooth domain in order to work with the Sobolev spaces that are the closure of indefinitely differentiable and null functions on the bounary, and to going back later to the classical Sobolev space. Then, we assume that the domain is extensible. Next, we obtain a priori estimates and convergence results in the approach problem, which allow us to delete the penalization term. To finish, we introduce a notion of entropy solution for our main problem and prove that it is the limit of the solution obtained in the variational case.
\end{abstract}

Keywords: Elliptic Problem, Entropy Solution, Nonlinear Boundary Conditions, Graph, Measure-data

\section{Introduction}

Consider the nonlinear elliptic equation

$$
\left\{\begin{array}{c}
u-\operatorname{div} a(u, \nabla u)=v \quad \text { in } \Omega \\
-\langle a(u, \nabla u), \eta\rangle \in \beta(x, u) \quad \text { on } \partial \Omega
\end{array}\right.
$$

where $\Omega$ is a smooth bounded domain in $\mathbb{R}^{N}$ with Lipschitz boundary $\partial \Omega$ and $1<\mathrm{p}<\mathrm{N}$,

$\eta$ is the unit outward normal vector on $\partial \Omega, v$ is a diffuse measure such that $v=v[\Omega$.

For a. e. $x \in \partial \Omega, \beta(x, r)=\partial j(x, r)$ is the subdifferential of a function $j: \partial \Omega \times \mathbb{R} \rightarrow[0, \infty]$ which is convex lower semicontinuous (1. s. c. for short) in $r \in \mathbb{R}$ for $\sigma$-a. e. $x \in \partial \Omega$, measurable with respect to the $(N-1)$-dimensionnal Hausdorff measure $\sigma$ on $\partial \Omega$ and such that $j(., 0)=0$. The vector-valued function $a: \mathbb{R} \times \mathbb{R}^{N} \rightarrow \mathbb{R}$ is continuous satisfying the following classical Leray-Lions-type conditions:

$$
\begin{aligned}
& \left(H_{1}\right) \text {-Monotonicity in } \xi \in \mathbb{R}^{N}: \\
& \quad(a(r, \xi)-a(r, \eta)) .(\xi-\eta) \geq 0 \forall r \in \mathbb{R}, \forall \xi, \eta \in \mathbb{R}^{N} \\
& \left(H_{2}\right) \text {-Coerciveness: } \exists \lambda_{0}>0 \text { such that } \\
& \quad(a(r, \xi)-a(r, 0)) . \xi \geq \lambda_{0}|\xi|^{p} \forall r \in \mathbb{R}, \forall \xi \in \mathbb{R}^{N}
\end{aligned}
$$

$\left(H_{3}\right)-$ Growth restriction: there exists a continuous function $\Lambda: \mathbb{R}^{+} \rightarrow \mathbb{R}^{+}$such that

$$
|a(r, \xi)| \leq \Lambda(|r|)\left(1+|\xi|^{p-1}\right) \forall r \in \mathbb{R}, \forall \xi \in \mathbb{R}^{N}
$$

$\left(H_{4}\right)$-There exists $C: \mathbb{R} \times \mathbb{R} \rightarrow \mathbb{R}^{+}$continuous such that

$$
\begin{aligned}
|a(r, \xi)-a(r, \xi)| & \leq \mathrm{C}(r, s)|r-s|\left(1+|\xi|^{p-1}\right) \forall r, s \\
& \in \mathbb{R}, \forall \xi \in \mathbb{R}^{N}
\end{aligned}
$$

A typical example of a function $a$ satisfying theses hypotheses is $a(r, \xi)=|\xi|^{p-2} \xi+F(r)$, where $F: \mathbb{R} \rightarrow \mathbb{R}^{N}$ 
is a locally Lipschitz function.

Many results are known for elliptic problems in the variational setting for Dirichlet or Dirichlet-Neumann problems [1, 11, 16, 21-22, 24]. In the $L^{1}-$ setting, for elliptic and parabolic equations in divergence form, the new equivalent notions of entropy and renormalized solutions have been introduced. [2, 3, 9, 12]. Sbihi and Wittbold [22] used and extended the methods introduced by Andreu et al [3] to study the problem

$$
\left\{\begin{array}{c}
u-\operatorname{div} a(u, \nabla u)=f \quad \text { in } \Omega \\
-\langle a(u, \nabla u), \eta\rangle \in \beta(x, u) \quad \text { on } \partial \Omega
\end{array}\right.
$$

where $a$ is a divergentiel operator depending of $u$ and $\beta$ depending also on the space variable $x$, and $f \in L^{1}(\Omega)$.

In the present paper, we use and extend the methods introduced by Andreu et al [3] and by Sbihi and Wittbold [22], to study the problem

$$
\left\{\begin{array}{c}
u-\operatorname{div} a(u, \nabla u)=v \quad \text { in } \Omega \\
-\langle a(u, \nabla u), \eta\rangle \in \beta(x, u) \quad \text { on } \partial \Omega,
\end{array}\right.
$$

where $v$ is a diffuse measure such that $v=v[\Omega$.

We prove the existence of an entropy solution via the approximation method. The uniqueness of the solution is obtained by using the comparison principle. The main difficulty is that, when one uses the integration by parts formula in the variational approach (see section 3 below), it appear at the boundary, for the part of the measure-data $v$ which is in $W^{1, p}(\Omega)$, a term which cannot vanish. In order to treat this difficulty, we consider a smooth domain $\Omega$ in order to work with the space $W_{0}^{1, p}(\Omega)$ and to going back later to the space $W^{1, p}(\Omega)$. More precisely, $\Omega$ is assumed to be a bounded domain in $\mathbb{R}^{N}$ with a boundary $\partial \Omega$ of class $C^{1}$. Then, $\Omega$ is an extension domain (see [15]), so we can fix an open bounded subset $U_{\Omega}$ of $\mathbb{R}^{N}$ such that $\bar{\Omega} \subset U_{\Omega}$, and there exists a bounded linear operator

$$
E: W^{1, p}(\Omega) \rightarrow W_{0}^{1, p}\left(U_{\Omega}\right)
$$

for which

$$
E(u)=u \text { a. e. in } \Omega \text { for each } u \in W^{1, p}(\Omega)
$$

$\|E(u)\|_{W_{0}^{1, p}\left(U_{\Omega}\right)} \leq c\|u\|_{W^{1, p}(\Omega)}$, where $c$ is a constant depending only on $\Omega$.

We define

$$
\mathfrak{M}_{b}^{p}(\Omega):=\left\{v \in \mathcal{M}_{b}^{p}\left(U_{\Omega}\right): v \text { is concentrated on } \Omega\right\}
$$

This definition is independent of the open set $U_{\Omega}$. Note that for $u \in W^{1, p}(\Omega) \cap L^{\infty}(\Omega)$ and $v \in \mathfrak{M}_{b}^{p}(\Omega)$, we have

$$
\langle v, E(u)\rangle=\int_{\Omega} u d v
$$

On the other hand, as $v$ is diffuse (see [12]), there exists $f \in L^{1}\left(U_{\Omega}\right)$ and $F \in\left(L^{p \prime}\left(U_{\Omega}\right)\right)^{N}$ such that $v=f-\operatorname{div}(F)$ in $\mathcal{D}^{\prime}\left(U_{\Omega}\right)$. Therefore, we can also write

$$
\langle v, E(u)\rangle=\int_{U_{\Omega}} f E(u) d x+\int_{U_{\Omega}} F \cdot \nabla E(u) d x
$$

The rest of the paper is organized as follows. In the next section we make precise the notations which will be used in the sequel and recall some facts on measures and capacities. In section 3 , we study the problem (1) by variational methods. We introduce an accretive operator $A_{\delta}$ related to problem (1) and show that $R\left(I+\alpha A_{\delta}\right) \supset L^{\infty}(\Omega)$ for all $\alpha>0$. In section 4 , we introduce the notion of entropy solution and prove the existence and uniqueness of this solution. In order to do this, we characterize $\mathcal{A}$, the limit of the operator $A_{\delta}$ in $\mathcal{M}_{b}^{p}\left(U_{\Omega}\right)$.

\section{Preliminaries}

In this section, we introduce some notations and definitions used in this paper. We denote $|$.$| and d \sigma$ the $N$-dimensional Lebesgue measure in $\mathbb{R}^{N}$ and the $(N-$ 1) -dimensional Hausdorff measure of $\partial \Omega$, respectively. The norm in $L^{p}(\Omega)$ is denoted by $\|.\|_{p}, 1 \leq p \leq \infty$. $W^{1, p}(\Omega)$ denotes the classicals sobolev space endowed with the usual norm denoted $\|.\|_{1, p}$. It is wellknown that if $u \in W^{1, p}(\Omega)$, it is possible to define the trace of $u$ on $\partial \Omega$, where the continuous linear trace operator $\tau: W^{1, p}(\Omega) \rightarrow$ $W^{-\frac{1}{p^{\prime}} p}(\partial \Omega)$ is surjective $[19,20]$. For $0<q<\infty, \mathcal{M}^{q}(\Omega)$ is the Marcinkiewicz space defined as the set of measurable functions $g: \Omega \rightarrow \mathbb{R}$ such that

$$
|\{x \in \Omega:|g(x)|>k\}| \leq c k^{-q}, c<\infty(c f .[8])
$$

As usual, for $k>0$, we denote by $T_{k}$, the truncation function at height $k \geq 0$ defined by

$$
T_{k}(u)=\min \{k, \max \{u,-k\}\}=\left\{\begin{array}{c}
-k \text { if } u<-k \\
u \text { if }|u| \leq k \\
k \text { if } u>k
\end{array}\right.
$$

Let $\gamma$ be be a maximal monotone operator defined on $\mathbb{R}$. We recall the definition of the main section $\gamma_{0}$ of $\gamma$ :

$$
\gamma_{0}(s)
$$

$=\left\{\begin{array}{c}\text { the element of minimal absolute value of } \gamma(s) \text { if } \gamma(s) \neq \emptyset \\ +\infty \text { if }[s,+\infty) \cap D(\gamma)=\emptyset \\ -\infty \text { if }(-\infty, s] \cap D(\gamma)=\emptyset .\end{array}\right.$

We denote by $\bar{u}$ the average of $u$, i.e. $\bar{u}=\frac{1}{|\Omega|} \int_{\Omega} u(x) d x$. We note

$$
\mathcal{P}=\left\{\begin{array}{c}
S \in C^{1}(\mathbb{R}): S(0)=0,0 \leq S^{\prime} \\
\leq 1, \operatorname{supp}\left(S^{\prime}\right) \text { is compact }
\end{array}\right\}
$$

Let $\mathcal{B}$ be a multi-valued operator in $L^{1}(\Omega)$. Recall that $\mathcal{B}$ is said to be accretive in $L^{1}(\Omega)$ if

$$
\begin{gathered}
\|u-\tilde{u}\|_{1} \leq\|u-\tilde{u}+\alpha(v-\tilde{v})\|_{1} \text { for any }(u, v),(\tilde{u}, \tilde{v}) \\
\in \mathcal{B} ; \alpha>0
\end{gathered}
$$


$\mathcal{B}$ is called $T$-accretive if

$$
\left\|(u-\tilde{u})^{+}\right\|_{1} \leq\left\|(u-\tilde{u}+\alpha(v-\tilde{v}))^{+}\right\|_{1} \text { for any }(u, v),(\tilde{u}, \tilde{v}) \in \mathcal{B} ; \alpha>0
$$

Finally, $\mathcal{B}$ is called $m$-accretive (resp. $m-T$-accretive) in $L^{1}(\Omega)$ if $\mathcal{B}$ is accretive ( $T$-accretive) and moreover, $R(I+\alpha \mathcal{B})=L^{1}(\Omega)$ for any $\alpha>0$ (for more details about accretive operators and nonlinear semigroups, cf. $[5,6,10])$.

Now, let us introduce some notations and recall some facts about capacities and measures usued throughout this paper $[13,14,18]$. Let $G$ be an arbitrary fixed bounded open subset of $\mathbb{R}^{N}$ with $\bar{\Omega} \subset G$. Given a compact subset $K \subseteq G$, we define the $p$ - capacity of $K$ by:

$$
C_{1, p}(K):=\inf \left\{\|\varphi\|_{1, p} ; \varphi \in C_{c}^{\infty}(G), \varphi \geq \chi_{K}\right\}
$$
by

The $p$-capacity of an arbitrary subset $E \subseteq G$ is defined

$$
C_{1, p}(E):=\inf \left\{C_{1, p}(O) ; O \text { open }, E \subseteq O\right\}
$$

As usual, a property will be said to hold cap-quasi everywhere (q. e. for short) if it holds everywhere except on a set of zero capacity.

Let $\mathcal{M}_{b}(\Omega)$ (resp. $\mathcal{M}_{b}(\partial \Omega)$ ) be the space of all Radon measures on $\Omega$ (resp. $\partial \Omega$ ) with bounded total variation. For $\mu \in \mathcal{M}_{b}(\partial \Omega)$, denote by $\mu^{+}, \mu^{-}$and $|\mu|$ the positive part, negative part and the total variation of the measure $\mu$, respectively, and denote by $\mu=\mu_{r} d \sigma+\mu_{s}$ the Radon-Nikodym decomposition of $\mu$ relatively to the $(N-1)$-dimensional Hausdorff measure $d \sigma$. We denote by $\mathcal{M}_{b}^{p}(\Omega)$ (resp. $\mathcal{M}_{b}^{p}(\partial \Omega)$ ) the set of Radon measures $\mu$ which satisfy $\mu(B)=0$ for every Borel set $B \subseteq \Omega$ (resp. $B \subseteq \partial \Omega$ ) such that $C_{1, p}(B)=0$, i.e. the Radon measures which do not charge sets of 0 -capacity. We denote $\mathcal{J}_{0}(\partial \Omega)=\{j ; j: \partial \Omega \times$ $\mathbb{R} \rightarrow[0,+\infty]$, such that $\mathrm{j}(., \mathrm{r})$ is $\sigma-$ measurable $\forall \mathrm{r} \in$ $\mathbb{R}$ and $\mathrm{j}(\mathrm{x},$.$) is convex, l. s. c. satisfying j(x,)=$. 0 for a. e. $x \in \partial \Omega$ \}. For a.e. $x \in \partial \Omega$ we define

$$
\begin{aligned}
& \mathcal{J}: W^{\frac{1}{p^{\prime}}, p}(\partial \Omega) \cap L^{\infty}(\partial \Omega) \rightarrow[0, \infty] \\
& u \quad \mapsto \int_{\partial \Omega} j(., u) d \sigma .
\end{aligned}
$$

Note that $\mathcal{J}$ naturally extends to a functional $\hat{\mathcal{J}}$ on $W_{0}^{1, p}(G) \cap L^{\infty}(G)$ as follows: $\hat{\jmath}(u)=\int_{\partial \Omega} j(., \tau(u)) d \sigma$ for any $u \in W_{0}^{1, p}(G)$. We recall that the closure of $D(\hat{\mathcal{J}})$ in $W_{0}^{1, p}(G)$ is a convex bilateral set, so according to Andreianov and Bouhssis [4], there exist unique (in the sense q. e.) functions $\gamma_{+}, \gamma_{-}$which are cap-quasi-l. s. c. and cap-quasi-u. s. c. respectively, such that $\overline{D(\mathcal{J})} \|^{\|\cdot\|_{1}, p}=$ $\left\{u \in W^{\frac{1}{p^{\prime \prime}, p}}(\partial \Omega) ; \gamma_{-}(x) \leq \tilde{u}(x) \leq \gamma_{+}(x)\right.$ q.e.on $\left.\partial \Omega\right\}$.

Moreover $\gamma_{-}(x)=\inf _{n} \tilde{u}_{n}(x)=\lim _{n} \inf _{1 \leq k \leq n} \tilde{u}_{k}(x)$ q.e. $x \in \partial \Omega$ (respectively the corresponding analogue for $\gamma_{+}$) for any $\|\cdot\|_{\frac{1}{p^{\prime}} p}$ - dense sequence $\left(u_{n}\right)_{n}$ in $D(\mathcal{J})$. We define the subdifferential operator:

$$
\begin{aligned}
& \partial \mathcal{J} \subseteq\left(W^{\frac{1}{p^{\prime \prime} p}}(\partial \Omega) \cap L^{\infty}(\partial \Omega)\right) \\
& \times\left(W^{\frac{-1}{p^{\prime}}, p^{\prime}}(\partial \Omega)+\left(L^{\infty}(\partial \Omega)\right)^{*}\right)
\end{aligned}
$$

by

$$
\begin{gathered}
\mu \in \partial \mathcal{J}(u) \\
\Leftrightarrow\left\{\begin{array}{c}
u \in W^{\frac{1}{p^{\prime}, p}}(\partial \Omega) \cap L^{\infty}(\partial \Omega), \mu \in W^{\frac{-1}{p^{\prime}}, p^{\prime}}(\partial \Omega)+\left(L^{\infty}(\partial \Omega)\right)^{*} \\
\text { and } \mathcal{J}(w) \geq \mathcal{J}(u)+\langle\mu, w-u\rangle \forall w \in W^{\frac{1}{p^{\prime \prime}}}(\partial \Omega) \cap L^{\infty}(\partial \Omega),
\end{array}\right.
\end{gathered}
$$

where, here as in the following, if note explicitly stated otherwise, $\langle.,$.$\rangle denotes the duality between W^{\frac{1}{p^{\prime}}, p}(\partial \Omega) \cap$ $L^{\infty}(\partial \Omega)$ and its dual.

\section{Variational Approach}

Let $\Omega$ be a bounded domain in $\mathbb{R}^{N}$ with Lipschitz boundary, $\quad 1<p<N, a: \Omega \times \mathbb{R}^{N} \rightarrow \mathbb{R}^{N} \quad$ a mapping satisfying the assumptions $\left(H_{1}\right)-\left(H_{4}\right)$ and $\beta$ is such that $\beta(x,)=.\partial j(x,$.$) a. e. on \partial \Omega$, where $j \in \mathcal{J}_{0}(\partial \Omega)$.

To apply the classical variational approach, we need an $L^{\infty}$ - estimate on $u$, which is not evident to obtain directly in our problem. The obstacle which we encounter is that we can not get rid of term with $a(u, 0)$. To overcome this difficulty, following Sbihi and Wittbold [22], we first redefine and extend the function $\Lambda$ which appears in hypothesis $\left(H_{3}\right)$, on an odd monotone function $\psi$ on $\mathbb{R}$ such that $\left|\frac{a(k, 0)}{\psi(k)}\right| \rightarrow 0$ as $k \rightarrow \infty$. This will be possible by setting $\Lambda(r):=\sup _{|z| \leq r}\{\psi(|z|),|z||a(z, 0)|\}$ for $r \geq 0$. Secondly, we add a penalization term $\delta \psi(u)$ on the boundary for a fixed $\delta$. This allows us to compensate the term with $a(u, 0)$ by choosing $\mathrm{k}$ sufficient large such that $\left|\frac{a(k, 0)}{\psi(k)}\right|<\delta$.

In the next section, we tend $\delta$ to zero and the penalization term disappears. Consequently we obtain the entropy solution of our initial problem (1).

Now, we define the operator $A_{\delta}$ as follows:

$$
(u, v) \in A_{\delta} \text { if and only if } u \in W^{1, p}(\Omega) \cap L^{\infty}(\Omega) ;
$$

$$
v \in \mathfrak{M}_{b}^{p}(\Omega)
$$

and there exists a measure $\mu \in \mathcal{M}_{b}^{p}(\partial \Omega)$ with

$$
\mu_{r}(x) \in \partial j(x, u(x))+\partial I_{\left[\gamma_{-}(x), \gamma_{+}(x)\right]}(u(x)) \text { a. e. } x \in \partial \Omega
$$

such that for all $\phi \in W^{1, p}(\Omega) \cap L^{\infty}(\Omega)$

$$
\int_{\Omega} a(u, \nabla u) \cdot \nabla(u-\phi) d x
$$




$$
\begin{gathered}
+\delta \int_{\partial \Omega} \psi(u)(u-\phi) d \sigma \leq \int_{\Omega}(u-\phi) d v-\int_{\partial \Omega}(\tilde{u}-\tilde{\phi}) d \mu, \\
\tilde{u}=\gamma_{+} \mu_{s}^{+}-\text {a.e.on } \partial \Omega, \tilde{u}=\gamma_{-} \mu_{s}^{-}-\text {a.e. on } \partial \Omega,
\end{gathered}
$$

where for given interval $[a, b] \subset \mathbb{R}, I_{[a, b]}$ denotes the convex 1. s. c. functional on $\mathbb{R}$ defined by 0 on $[a, b],+\infty$ otherwise.

Our first main result is the following.

Theorem 1. The operator $\mathrm{A} \delta$ satisfies the following properties:

i) $\mathrm{A} \delta$ is $\mathrm{T}$-accretive in $\mathrm{L} 1(\Omega)$,

ii) $\mathrm{L} \infty(\Omega) \subset \mathrm{R}(\mathrm{I}+\alpha \mathrm{A} \delta)$ for any $\alpha>0$,

iii) $\mathrm{D}(\mathrm{A} \delta)$ is dense in $\mathrm{L} 1(\Omega)$.

Proof.

$$
\begin{gathered}
\frac{1}{k} \int_{\left\{(u-v)^{+}<k\right\}}[a(u, \nabla u)-a(v, \nabla v)] \cdot \nabla(u-v)^{+} d x+\frac{1}{k} \delta \int_{\partial \Omega}(\psi(u)-\psi(v)) T_{k}(u-v)^{+} d \sigma \\
\leq \frac{1}{k} \int_{\Omega}(v-u) T_{k}(u-v)^{+} d x+\frac{1}{k} \int_{\Omega} T_{k}(u-v)^{+} d v_{1}-\frac{1}{k} \int_{\Omega} T_{k}(u-v)^{+} d v_{2} \\
-\frac{1}{k}\left(\int_{\partial \Omega} T_{k}(\tilde{u}-\tilde{v})^{+} d \mu_{1}-\int_{\partial \Omega} T_{k}(\tilde{u}-\tilde{v})^{+} d \mu_{2}\right) .
\end{gathered}
$$

Denote by $I 1$ respectively $I 2$ the first, respectively the second integral in the left hand side of (5). Using hypothesis $(H 1)$, (H4) and the Lebesgue dominated convergence theorem, we obtain

$$
\begin{aligned}
& I_{1} \geq \quad \frac{1}{k} \int_{\left\{(u-v)^{+}<k\right\}}[a(u, \nabla v)-a(v, \nabla v)] \cdot \nabla(u-v)^{+} d x \\
& \geq-\frac{1}{k} \int_{\left\{(u-v)^{+}<k\right\}} C(u, v)(u-v)^{+}\left(1+|\nabla v|^{p-1}\right)\left|\nabla(u-v)^{+}\right| d x \\
& \geq \quad-\frac{C_{1} k}{k} \int_{\left\{(u-v)^{+}<k\right\}}\left(1+|\nabla v|^{p-1}\right)\left|\nabla(u-v)^{+}\right| d x \\
& \geq-C_{1} \int_{\Omega}\left(1+|\nabla v|^{p-1}\right)\left|\nabla(u-v)^{+}\right| \chi_{\left\{(u-v)^{+}<k\right\}} d x \rightarrow 0 \text { as } k \rightarrow 0 .
\end{aligned}
$$

Note that the properties of the measures $\mu 1$ and $\mu 2$ ensure that the second term in the brackets in the right hand side of (5) is nonnegative. Indeed, these integrals can be written as

$$
\begin{gathered}
\int_{\partial \Omega} T_{k}(\tilde{u}-\tilde{v})^{+}\left(\mu_{r, 1}-\mu_{r, 2}\right)+\int_{\partial \Omega} T_{k}\left(\gamma_{+}-\tilde{v}\right) d\left(\mu_{s, 1}\right)^{+} \\
-\int_{\partial \Omega} T_{k}\left(-\gamma_{+}+\tilde{u}\right) d\left(\mu_{s, 2}\right)^{+}-\int_{\partial \Omega} T_{k}\left(\gamma_{-}-\tilde{v}\right) d\left(\mu_{s, 1}\right)^{-}-\int_{\partial \Omega} T_{k}\left(-\gamma_{-}+\tilde{u}\right) d\left(\mu_{s, 2}\right)^{-}
\end{gathered}
$$

which are, cleary, nonnegative by properties of $\mu_{1}, \mu_{2}$ and $\gamma_{+/-}$. Thanks to the monotonicity of $\psi$, we have

$$
I 2 \geq 0 \text {. }
$$

We can write for the second term in the right hand side of (5),

$$
\begin{array}{rlc}
\frac{1}{k} \int_{\Omega} T_{k}(u-v)^{+} d v_{1} & = & \int_{\Omega} E\left(\frac{1}{k} T_{k}(u-v)^{+}\right) d v_{1} \\
& = & \left\langle v_{1}, E\left(\frac{1}{k} T_{k}(u-v)^{+}\right)\right\rangle \\
& = & \int_{\Omega} f \frac{1}{k} T_{k}(u-v)^{+} d x+\int_{U_{\Omega}} F_{1} \cdot \nabla E\left(\chi_{\Omega} \frac{1}{k} T_{k}(u-v)^{+}\right) d x
\end{array}
$$

As $\left|\chi_{\Omega} \frac{1}{k} T_{k}(u-v)^{+}\right| \leq 1$ for all $k>0$, then we use the inequality

$$
\|E(v)\|_{W_{0}^{1, p}\left(U_{\Omega}\right)} \leq c\|v\|_{W^{1, p}(\Omega)}, \forall v \in W^{1, p}(\Omega)
$$

to conclude that the sequence $\left(E\left(\frac{1}{k} T_{k}(u-v)^{+}\right)\right)_{k>0}$ is uniformly bounded in $W_{0}^{1, p}\left(U_{\Omega}\right)$. We also have $E\left(\chi_{\Omega} \frac{1}{k} T_{k}(u-v)^{+}\right) \rightarrow \operatorname{sign}_{0}^{+}$a. e. in $U_{\Omega}$ as $k \rightarrow 0$.
Then,

$$
\nabla E\left(\chi_{\Omega} \frac{1}{k} T_{k}(u-v)^{+}\right)-0 \text { in }\left(L^{p}\left(U_{\Omega}\right)\right)^{N}
$$

Finally, we get for the second term in the right hand side of (8),

$$
\lim _{k \rightarrow 0} \int_{U_{\Omega}} F_{1} \cdot \nabla E\left(\chi_{\Omega} \frac{1}{k} T_{k}(u-v)^{+}\right) d x=0
$$

By the same manage, we get 
$\frac{1}{k} \int_{\Omega} T_{k}(u-v)^{+} d v_{2}=$
$\int_{\Omega} g \frac{1}{k} T_{k}(u-v)^{+} d x+\int_{U_{\Omega}} F_{2} \cdot \nabla E\left(\chi_{\Omega} \frac{1}{k} T_{k}(u-v)^{+}\right) d x$

and

$$
\lim _{k \rightarrow 0} \int_{U_{\Omega}} F_{2} \cdot \nabla E\left(\chi_{\Omega} \frac{1}{k} T_{k}(u-v)^{+}\right) d x=0
$$

Combining (6)-(11) and using Lebesgue dominated convergence theorem, we can pass to the limit in (5) with $k \rightarrow 0$ to get

$$
\begin{aligned}
& \lim _{k \rightarrow 0} \frac{1}{k} \int_{\Omega}(u-v) T_{k}(u-v)^{+} d x \\
\leq & \lim _{k \rightarrow 0} \frac{1}{k} \int_{\Omega}(f-g) T_{k}(u-v)^{+} d x,
\end{aligned}
$$

which implies

$$
\int_{\Omega}(u-v)^{+} d x \leq \int_{\Omega}(f-g)^{+} d x .
$$

Consequently (4) holds.

ii) It will be no restriction to assume that $\alpha=1$. In order to

$$
\begin{gathered}
\left\langle A_{\delta, \lambda} u_{\lambda}, \phi\right\rangle=\int_{\Omega} T_{l}\left(u_{\lambda}\right) \phi d x+\lambda \int_{\Omega}\left|T_{l}\left(u_{\lambda}\right)\right|^{p-2} T_{l}\left(u_{\lambda}\right) \phi d x+\int_{\Omega} a\left(T_{l}\left(u_{\lambda}\right), \nabla u_{\lambda}\right) . \nabla \phi d x \\
+\int_{\partial \Omega} \beta_{\lambda}\left(., T_{l}\left(u_{\lambda}\right)\right) \phi d \sigma+\delta \int_{\partial \Omega} T_{l}\left(\psi\left(u_{\lambda}\right)\right) \phi d \sigma
\end{gathered}
$$

for all $\phi \in W^{1, p}(\Omega)$. Here, $\langle.,$.$\rangle denotes the duality pairing$ between $W^{1, p}(\Omega)$ and $\left[W^{1, p}(\Omega)\right]^{*}$. We have the following results.

Lemma 1. (see [22], Lemma 3.1) The operator $A_{\delta, \lambda}$ is bounded, coercive and verifies the (M)-property.

Lemma 2. (cf. [23]) Let $X$ be a reflexive Banach space and

$\mathrm{A}: \mathrm{X} \rightarrow \mathrm{X}^{\prime}$ an operator such that

(i) A is bounded,

(ii) $\mathrm{A}$ is coercive,

(iii) $\mathrm{A}$ is of the type (M),

then $\mathrm{A}$ is surjective.

By Lemma 2 , the operator $A_{\delta, \lambda}$ is surjective. So, for all $v_{\lambda} \in\left[W^{1, p}(\Omega)\right]^{*}$ there exists $u_{\lambda} \in W^{1, p}(\Omega)$ such that for all prove that $L^{\infty}(\Omega) \subset R\left(I+A_{\delta}\right)$, we regularize $v$ as follows: $\forall \lambda>0, \forall x \in U_{\Omega}$ we define

$$
f_{\lambda}(x)=T_{\lambda}(f(x)) \chi_{\Omega}(x)
$$

Let $\left(F_{\lambda}\right)_{\lambda \geq 1} \subset C_{0}^{\infty}\left(U_{\Omega}\right)$ be a sequence such that $F_{\lambda} \rightarrow F$ strongly in $\left(L^{p^{\prime}}\left(U_{\Omega}\right)\right)^{N}$. For any $\lambda \geq 1$ we set $\tilde{F}_{\lambda}=$ $\chi_{\Omega} F_{\lambda}$ and $v_{\lambda}=f_{\lambda}-\operatorname{div}\left(\tilde{F}_{\lambda}\right)$. For any $n \geq 1$, one has $v_{\lambda} \in \mathfrak{M}_{b}^{p}(\Omega), v_{\lambda} \rightarrow v$ in $\mathcal{M}_{b}\left(U_{\Omega}\right)$ and $v_{\lambda} \in L^{\infty}(\Omega)$. Therefore, we approximate the problem (1.1) by equations of the form

$\left\{\begin{array}{c}T_{l}\left(u_{\lambda}\right)+\lambda\left|T_{l}\left(u_{\lambda}\right)\right|^{p-2} T_{l}\left(u_{\lambda}\right)-\operatorname{div} a\left(T_{l}\left(u_{\lambda}\right), \nabla u_{\lambda}\right)=v_{\lambda} \text { in } \Omega, \\ -a\left(T_{l}\left(u_{\lambda}\right), \nabla u_{\lambda}\right) \cdot \eta=\beta_{\lambda}\left(x, T_{l}\left(u_{\lambda}\right)\right)+\delta T_{l}\left(\psi\left(u_{\lambda}\right)\right) \text { on } \partial \Omega\end{array}\right.$

Where $k \geq\left\|v_{\lambda}\right\|_{\infty}+1$ satisfies $\left|\frac{a(k, 0)}{\psi(k)}\right|<\delta$, with $l>\max \{k, \psi(k)\}$.

Here for every $\lambda \in \mathbb{N}^{*}, \beta_{\lambda}(x,$.$) is the Yosida$ approximation of $\beta(x$, . $)$, i.e.

$$
\beta_{\lambda}(x, .)=\lambda\left(I-\left(I+\frac{1}{\lambda} \beta(x, .)\right)\right)^{-1}
$$

Consider the operator $A_{\delta, \lambda}: W^{1, p}(\Omega) \rightarrow\left[W^{1, p}(\Omega)\right]^{*}$ defined by:

$$
\begin{aligned}
& \int_{\Omega} T_{l}\left(u_{\lambda}\right) p_{\varepsilon}^{+}\left(u_{\lambda}-k\right) d x+\lambda \int_{\Omega}\left|u_{\lambda}\right|^{p-2} u_{\lambda} p_{\varepsilon}^{+}\left(u_{\lambda}-k\right) d x+\frac{1}{\varepsilon} \int_{\left\{k<u_{\lambda}<k+\varepsilon\right\}} a\left(T_{l}\left(u_{\lambda}\right), 0\right) . \nabla u_{\lambda} d x \\
& \leq \int_{\Omega} p_{\varepsilon}^{+}\left(u_{\lambda}-k\right) d v_{\lambda}-\delta \int_{\partial \Omega} T_{l}\left(\psi\left(u_{\lambda}\right)\right) p_{\varepsilon}^{+}\left(u_{\lambda}-k\right) d \sigma-\int_{\partial \Omega} \beta_{\lambda}\left(., T_{l}\left(u_{\lambda}\right)\right) p_{\varepsilon}^{+}\left(u_{\lambda}-k\right) d \sigma .
\end{aligned}
$$

Note that, since $l>k$ and by Green formula, we have

$$
\begin{aligned}
\lim _{\varepsilon \rightarrow 0} \inf \frac{1}{\varepsilon} \int_{\left\{k<u_{\lambda}<k+\varepsilon\right\}} a\left(T_{l}\left(u_{\lambda}\right), 0\right) . \nabla u_{\lambda} d x & \geq \quad-|a(k, 0)| \int_{\partial \Omega \cap\left\{u_{\lambda}>k\right\}} d \sigma \\
& \geq-|a(k, 0)| \int_{\partial \Omega \cap\left\{u_{\lambda}>k\right\}} \frac{T_{l}\left(\psi\left(u_{\lambda}\right)\right)}{T_{l}(\psi(k))} d \sigma \\
& \geq \frac{-|a(k, 0)|}{T_{l}(\psi(k))} \int_{\partial \Omega \cap\left\{u_{\lambda}>k\right\}} T_{l}\left(\psi\left(u_{\lambda}\right)\right) d \sigma \\
\geq & -\delta \int_{\partial \Omega \cap\left\{u_{\lambda}>k\right\}} T_{l}\left(\psi\left(u_{\lambda}\right)\right) d \sigma .
\end{aligned}
$$


We also have

$$
\begin{gathered}
\int_{\Omega} p_{\varepsilon}^{+}\left(u_{\lambda}-k\right) d v_{\lambda}=\int_{\Omega} E\left(p_{\varepsilon}^{+}\left(u_{\lambda}-k\right)\right) d v_{\lambda} \\
=\left\langle v_{\lambda}, E\left(p_{\varepsilon}^{+}\left(u_{\lambda}-k\right)\right)\right\rangle \\
=\int_{\Omega} T_{\lambda}(f) p_{\varepsilon}^{+}\left(u_{\lambda}-k\right) d x+\int_{U_{\Omega}} F_{\lambda} \cdot \nabla E\left(\chi_{\Omega} p_{\varepsilon}^{+}\left(u_{\lambda}-k\right)\right) d x
\end{gathered}
$$

By the Lebesgue dominated convergence theorem, we have for the first term of the right hand side of (14),

$$
\lim _{\varepsilon \rightarrow 0} \int_{\Omega} T_{\lambda}(f) p_{\varepsilon}^{+}\left(u_{\lambda}-k\right) d x=\int_{\left\{u_{\lambda}>k\right\}} f_{\lambda} d x
$$

As $\left|\chi_{\Omega} p_{\varepsilon}^{+}\left(u_{\lambda}-k\right)\right| \leq 1$ for all $\varepsilon>0$, then we use the inequality

$$
\|E(v)\|_{W_{0}^{1, p}\left(U_{\Omega}\right)} \leq c\|v\|_{W^{1, p}(\Omega)}, \forall v \in W^{1, p}(\Omega)
$$

to conclude that the sequence $\left(E\left(p_{\varepsilon}^{+}\left(u_{\lambda}-k\right)\right)\right)_{\varepsilon>0}$ is uniformly bounded in $W_{0}^{1, p}\left(U_{\Omega}\right)$. We also have $E\left(p_{\varepsilon}^{+}\left(u_{\lambda}-\right.\right.$ $k)) \rightarrow \chi_{\left\{u_{\lambda}>k\right\}}$ a. e. in $U_{\Omega}$ as $\varepsilon \rightarrow 0$. We also have

$\nabla E\left(p_{\varepsilon}^{+}\left(u_{\lambda}-k\right)\right) \rightarrow 0$ in $\left(L^{p}\left(U_{\Omega}\right)\right)^{N}$. Finally, we get for the second term in the right hand side of (14),

$$
\lim _{\varepsilon \rightarrow 0} \int_{U_{\Omega}} F_{\lambda} \cdot \nabla E\left(\chi_{\Omega} p_{\varepsilon}^{+}\left(u_{\lambda}-k\right)\right) d x=0
$$

Passing to the limit in (13) with $\varepsilon \rightarrow 0$, taking account (15)-(16) and have in mind that $\beta_{\lambda}\left(., T_{l}\left(u_{\lambda}\right)\right)$ and $\left|u_{\lambda}\right|^{p-2} u_{\lambda}$ are nonnegative in $\left\{u_{\lambda}>k\right\}$, we get

$$
\begin{gathered}
\int_{\left\{u_{\lambda}>k\right\}} T_{l}\left(u_{\lambda}\right) d x \leq \int_{\left\{u_{\lambda}>k\right\}} f_{\lambda} d x+\delta \int_{\partial \Omega \cap\left\{u_{\lambda}>k\right\}} T_{l}\left(\psi\left(u_{\lambda}\right)\right) d \sigma \\
-\delta \int_{\partial \Omega \cap\left\{u_{\lambda}>k\right\}} T_{l}\left(\psi\left(u_{\lambda}\right)\right) d \sigma \\
\leq \int_{\left\{u_{\lambda}>k\right\}} f_{\lambda} d x .
\end{gathered}
$$

Then

$$
\int_{\left\{u_{\lambda}>k\right\}}\left(T_{l}\left(u_{\lambda}\right)-T_{l}(k)\right) d x \leq \int_{\left\{u_{\lambda}>k\right\}}\left(f_{\lambda}-T_{l}(k)\right) d x
$$

As $l>k$ then $T_{l}(k)=k$. Thus, as $k \geq\left\|f_{\lambda}\right\|_{\infty}+1$ we have

$$
\int_{\left\{u_{\lambda}>k\right\}}\left(f_{\lambda}-T_{l}(k)\right) d x=\int_{\left\{u_{\lambda}>k\right\}}\left(f_{\lambda}-k\right) d x \leq 0
$$

From inequality above, we get

$$
\int_{\left\{u_{\lambda}>k\right\}}\left(T_{l}\left(u_{\lambda}\right)-T_{l}(k)\right)^{+} d x \leq 0, \forall l>k
$$

and then $T_{l}\left(u_{\lambda}\right) \leq k$ a. e. in $\left\{u_{\lambda}>k\right\}$. We conclude that

$$
u_{\lambda} \leq k \text { a.e.in } \Omega \text {. }
$$

Similarly, we prove that

$$
u_{\lambda} \geq-k \text { a.e.in } \Omega \text {. }
$$

Consequently,

$$
\left\|u_{\lambda}\right\|_{\infty} \leq C
$$

where $C$ is a constant depending on $v$.

Taking $\phi=0$ as a test function in (12), we get after using (H2)

$$
\begin{gathered}
\int_{\Omega} T_{l}\left(u_{\lambda}\right) u_{\lambda} d x+\lambda \int_{\Omega}\left|u_{\lambda}\right|^{p} d x+\lambda_{0} \int_{\Omega}\left|\nabla u_{\lambda}\right|^{p} d x+\int_{\Omega} a\left(T_{l}\left(u_{\lambda}\right), 0\right) . \nabla u_{\lambda} d x \\
+\int_{\partial \Omega} \beta_{\lambda}\left(., T_{l}\left(u_{\lambda}\right)\right) u_{\lambda} d \sigma+\delta \int_{\partial \Omega} T_{l}\left(\psi\left(u_{\lambda}\right)\right) u_{\lambda} d \sigma \\
\leq \int_{\Omega} u_{\lambda} d v_{\lambda} .
\end{gathered}
$$

By Gauss-Green formula, the hypothesis (H3) and (17), we deduce that

$$
\left|\int_{\Omega} a\left(T_{l}\left(u_{\lambda}\right), 0\right) . \nabla u_{\lambda}\right| \leq C .
$$

As $b, \beta \lambda$ and $T l \circ \psi$ are nondecreasing then, according to Young inequality, we get from (18):

$$
\begin{aligned}
\lambda_{0} \int_{\Omega}\left|\nabla u_{\lambda}\right|^{p} d x & \leq\left|\int_{\Omega} a\left(T_{l}\left(u_{\lambda}\right), 0\right) \cdot \nabla u_{\lambda} d x\right|+\int_{\Omega} u_{\lambda} d v_{\lambda} \\
& \leq C+C(v, \Omega)\left\|u_{\lambda}\right\|_{\infty}
\end{aligned}
$$

From (17) and (19), it follows that $\left(u_{\lambda}\right)_{\lambda}$ is uniformly bounded in $W^{1, p}(\Omega)$. Hence, there exists a subsequence still denoted $\left(u_{\lambda}\right)_{\lambda}, \quad$ such that $u_{\lambda} \rightarrow u$ weakly in $W^{1, p}(\Omega)$ as $\lambda \rightarrow+\infty \quad$. By Rellich-Kondrachov theorem, $\quad u_{\lambda} \rightarrow u$ in $L^{p}(\Omega) \quad$ and $\left(u_{\lambda}\right) \rightarrow \tau(u)$ in $L^{p}(\partial \Omega)$ as $\lambda \rightarrow+\infty$. Then $T_{l}\left(\psi\left(u_{\lambda}\right)\right) \rightarrow$ $\psi(u)$ on $\partial \Omega$. We may also assume that $u_{\lambda} \rightarrow u$ a. e. in $\Omega$. Therefore, by (17),

$$
\|u\|_{\infty} \leq C(v, \Omega)
$$

We have $\left|\beta_{\lambda}\left(., T_{l}\left(u_{\lambda}\right)\right)\right| \leq \beta_{\lambda}(., l)$, so

$$
\int_{\partial \Omega}\left|\beta_{\lambda}\left(., T_{l}\left(u_{\lambda}\right)\right)\right| d \sigma \leq C
$$

Thus, passing to a subsequence if necessary, we have $\beta_{\lambda}\left(., T_{l}\left(u_{\lambda}\right)\right) \rightarrow \mu$ weakly in $\mathcal{M}_{b}(\partial \Omega)$ as $\lambda \rightarrow+\infty$.

Note that for all $\lambda>v>0$, we have for a. e. $x \in$ $\partial \Omega,\left|\beta_{\lambda}(x, r)\right| \geq\left|\beta_{v}(x, r)\right|, \forall r \in \mathbb{R}$. Thus from (20), $\int_{\partial \Omega}\left|\beta_{\nu}\left(., T_{l}\left(u_{\lambda}\right)\right)\right| \leq C$. Passing to the limit with $\lambda \rightarrow+\infty$, we get $\int_{\partial \Omega}\left|\beta_{v}\left(., T_{l}(u)\right)\right| \leq C$. As $v \rightarrow+\infty$, we obtain $\int_{\partial \Omega}\left|\beta^{0}\left(., T_{l}(u)\right)\right| \leq C$. Here $\beta^{0}(., r)$ is the main section of 
$\beta(., r)$.

Next, thanks to (17), (19) and hypothesis $\left(H_{3}\right)$, we have

$$
\int_{\Omega}\left|a\left(u_{\lambda}, \nabla u_{\lambda}\right)\right|^{p^{\prime}} d x \leq C
$$

From (21), it follows that $\left(a\left(u_{\lambda}, \nabla u_{\lambda}\right)\right)_{\lambda}$ is uniformly bounded in $\left(L^{p \prime}(\Omega)\right)^{N}$. After passing to a suize subsequence, we can assume that $a\left(u_{\lambda}, \nabla u_{\lambda}\right) \rightarrow \chi$ weakly in
$\left(L^{p \prime}(\Omega)\right)^{N}$. as $\lambda \rightarrow+\infty$. The aim is to show, via a pseudo-monotonicity argument that $\operatorname{div}(a(u, \nabla u))=\operatorname{div} \chi$. To this end, we must show that

$$
\lim \sup _{\lambda \rightarrow+\infty} \int_{\Omega} a\left(u_{\lambda}, \nabla u_{\lambda}\right) \cdot \nabla\left(u_{\lambda}-u\right)=0
$$

Taking $\phi=u_{\lambda}-\left(u_{\lambda}-u\right)^{+}$as a test function in (12), we get

$$
\begin{gathered}
\int_{\Omega} a\left(u_{\lambda}, \nabla u_{\lambda}\right) \cdot \nabla\left(u_{\lambda}-u\right)^{+} \leq \int_{\Omega}\left(u_{\lambda}-u\right)^{+} d v_{\lambda}-\int_{\Omega} u_{\lambda}\left(u_{\lambda}-u\right)^{+} d x \\
-\lambda \int_{\Omega}\left|u_{\lambda}\right|^{p-2} u_{\lambda}\left(u_{\lambda}-u\right)^{+} d x-\int_{\partial \Omega} \beta_{\lambda}\left(., u_{\lambda}\right)\left(u_{\lambda}-u\right)^{+} d \sigma \\
-\delta \int_{\partial \Omega} T_{l}\left(\psi\left(u_{\lambda}\right)\right)\left(u_{\lambda}-u\right)^{+} d \sigma .
\end{gathered}
$$

We have $\beta_{\lambda}\left(., u_{\lambda}\right)=\beta_{\lambda}\left(., u_{\lambda}{ }^{+}\right)+\beta_{\lambda}\left(.,-u_{\lambda}{ }^{-}\right)$and $\beta_{\lambda}\left(., u_{\lambda}{ }^{+}\right)\left(u_{\lambda}-u\right)^{+} \geq 0$. Then, from inequality (23) we deduce

$$
\begin{gathered}
\int_{\Omega} a\left(u_{\lambda}, \nabla u_{\lambda}\right) \cdot \nabla\left(u_{\lambda}-u\right)^{+} \leq \int_{\Omega}\left(u_{\lambda}-u\right)^{+} d v_{\lambda}-\int_{\Omega} u_{\lambda}\left(u_{\lambda}-u\right)^{+} d x \\
-\lambda \int_{\Omega}\left|u_{\lambda}\right|^{p-2} u_{\lambda}\left(u_{\lambda}-u\right)^{+} d x-\int_{\partial \Omega} \beta_{\lambda}\left(.,-u_{\lambda}^{-}\right)\left(u_{\lambda}-u\right)^{+} d \sigma \\
-\delta \int_{\partial \Omega} T_{l}\left(\psi\left(u_{\lambda}\right)\right)\left(u_{\lambda}-u\right)^{+} d \sigma .
\end{gathered}
$$

Having in mind that $\left(u_{\lambda}\right)_{\lambda}$ is uniformly bounded in $L^{\infty}(\partial \Omega)$, we have $\left\|\left(u_{\lambda}-u\right)^{+}\right\|_{\infty} \leq C$ and $\left(u_{\lambda}-u\right)^{+} \rightarrow 0$ a. e., as $\lambda \rightarrow+\infty$.

Next, observe that $\quad \beta_{\lambda}\left(.,-u_{\lambda}^{-}\right) \geq \beta_{\lambda}\left(.,-u^{-}\right) \geq$ $\beta^{0}\left(.,-u^{-}\right)$on $\left\{u_{\lambda} \geq u\right\}$. As $\left|\beta^{0}\left(.,-u^{-}\right)\right| \in L^{1}(\partial \Omega)$, by Lebesgue dominated convergence theorem, it follows that $\int_{\partial \Omega} \beta_{\lambda}\left(.,-u_{\lambda}{ }^{-}\right)\left(u_{\lambda}-u\right)^{+} \rightarrow 0$, as $\rightarrow+\infty$. Moreover

$$
\begin{gathered}
\int_{\Omega}\left(u_{\lambda}-u\right)^{+} d v_{\lambda}=\int_{\Omega} E\left(\left(u_{\lambda}-u\right)^{+}\right) d v_{\lambda} \\
=\left\langle v_{\lambda}, E\left(\left(u_{\lambda}-u\right)^{+}\right)\right\rangle \\
=\int_{\Omega} T_{\lambda}(f)\left(u_{\lambda}-u\right)^{+} d x+\int_{U_{\Omega}} F_{\lambda} \cdot \nabla E\left(\chi_{\Omega}\left(u_{\lambda}-u\right)^{+}\right) d x
\end{gathered}
$$

By the Lebesgue dominated convergence theorem, we have for the first term of the right hand side of (25),

$$
\lim _{\lambda \rightarrow+\infty} \int_{\Omega} T_{\lambda}(f)\left(u_{\lambda}-u\right)^{+} d x=0
$$

As $\left|\chi_{\Omega}\left(u_{\lambda}-u\right)^{+}\right| \leq C(v, \Omega)$ for all $\lambda>0$, then we use the inequality

$$
\|E(v)\|_{W_{0}^{1, p}\left(U_{\Omega}\right)} \leq c\|v\|_{W^{1, p}(\Omega)}, \forall v \in W^{1, p}(\Omega)
$$

to conclude that the sequence $\left(E\left(u_{\lambda}-u\right)^{+}\right)_{\lambda>0}$ is uniformly bounded in $W_{0}^{1, p}\left(U_{\Omega}\right)$. We also have $E\left(u_{\lambda}-u\right)^{+} \rightarrow 0$ a. e. in $U_{\Omega}$ as $\lambda \rightarrow+\infty$. Then, $\nabla E\left(u_{\lambda}-u\right)^{+} \rightarrow 0$ in $\left(L^{p}\left(U_{\Omega}\right)\right)^{N}$.

Finally, we get for the second term in the right hand side of (25),

$$
\lim _{\lambda \rightarrow+\infty} \int_{U_{\Omega}} F_{\lambda} \cdot \nabla E\left(\chi_{\Omega}\left(u_{\lambda}-u\right)^{+}\right) d x=0
$$

The inequality

$$
\limsup _{\lambda \rightarrow+\infty} \int_{\Omega} a\left(u_{\lambda}, \nabla u_{\lambda}\right) \cdot \nabla\left(-\left(u_{\lambda}-u\right)^{-}\right) \leq 0
$$

follows similarly. Hence

$$
\limsup _{\lambda \rightarrow+\infty} \int_{\Omega} a\left(u_{\lambda}, \nabla u_{\lambda}\right) \cdot \nabla\left(u_{\lambda}-u\right) \leq 0
$$

and (22) follows from the monotonicity of $a$.

Now, let $\phi \in C_{c}\left(\mathbb{R}^{N}\right)$ and $\alpha \in \mathbb{R}^{*}$. Using the hypothesis (H1), the Lebesgue dominated convergence theorem and the relation (22), we get

$$
\begin{gathered}
\alpha \lim _{\lambda \rightarrow+\infty} \int_{\Omega}\left[a\left(u_{\lambda}, \nabla u_{\lambda}\right)-a(u, \nabla(u-\alpha \phi))\right] \cdot \nabla \phi d x \\
\geq \limsup _{\lambda \rightarrow+\infty} \int_{\Omega} a(u, \nabla(u-\alpha \phi)) \cdot \nabla\left(u_{\lambda}-u\right) d x \\
=0 .
\end{gathered}
$$

Dividing the quantity $\alpha \lim _{\lambda \rightarrow+\infty} \int_{\Omega}\left[a\left(u_{\lambda}, \nabla u_{\lambda}\right)-\right.$ $a(u, \nabla(u-\alpha \phi))] \cdot \nabla \phi d x$ by $\alpha>0$ and by $\alpha<0$ successively, and passing to limit with $\alpha \rightarrow 0$, we get

$$
\begin{aligned}
\lim _{\lambda \rightarrow+\infty} \int_{\Omega} a\left(u_{\lambda}, \nabla u_{\lambda}\right) \cdot \nabla \phi d x & =\lim _{\alpha \rightarrow 0} \int_{\Omega} a(u, \nabla(u-\alpha \phi)) \cdot \nabla \phi d x \\
& =\int_{\Omega} a(u, \nabla u) \cdot \nabla \phi d x,
\end{aligned}
$$

i.e. $a\left(u_{\lambda}, \nabla u_{\lambda}\right) \rightarrow a(u, \nabla u)$ weakly in $\left(L^{p \prime}(\Omega)\right)^{N}$. Hence $\operatorname{div}(a(u, \nabla u))=\operatorname{div} \chi$.

Up to now, we have shown that for all $\phi \in C_{c}\left(\mathbb{R}^{N}\right)$, passing to the limit in (12) with $\lambda \rightarrow+\infty$, we get

$$
\begin{aligned}
& \int_{\Omega} a(u, \nabla u) \cdot \nabla(u-\phi) d x+\delta \int_{\partial \Omega} \psi(u)(u-\phi) d \sigma \\
\leq & \int_{\Omega}(u-\phi) d v-\int_{\Omega} u(u-\phi) d x-\int_{\partial \Omega}(\tilde{u}-\tilde{\phi}) d \mu .
\end{aligned}
$$

By density, the last inequality remains true for all $\phi \in$ $W^{1, p}(\Omega) \cap L^{\infty}(\Omega)$. Then, we can conclude that

$$
\begin{gathered}
\int_{\Omega} a(u, \nabla u) \cdot \nabla \phi d x+\delta \int_{\partial \Omega} \psi(u) \phi d \sigma=\int_{\Omega} \phi d v- \\
\int_{\Omega} u \phi d x-\int_{\partial \Omega} \tilde{\phi} d \mu
\end{gathered}
$$

for all $\phi \in W^{1, p}(\Omega) \cap L^{\infty}(\Omega)$.

Finally, as Sbihi and Wittbold [22], we characterize the measure $\mu$ by proving that 


$$
J(\xi) \geq J(u)+\langle\mu, \xi-u\rangle, \forall \xi \in C(\partial \Omega)
$$

Since $\mu \in \mathcal{M}_{b}^{p}(\partial \Omega)$, one can say that the inequality (29) holds for $\xi \in W^{\frac{1}{p \prime}, p}(\partial \Omega) \cap L^{\infty}(\partial \Omega)$ and thus we deduce that $\mu \in \partial J(u)$.

To conclude the proof of ii), we prove, using the fact that $\mu \in \partial J(u)$ and same technics used by Bouchitté ([13], Proposition 20) that the measure $\mu$ satisfies

$$
\begin{gathered}
\mu_{r}(x) \in \partial j(x, u(x))+\partial I_{\left[\gamma_{-}(x), \gamma_{+}(x)\right]}(u(x)) \text { a. e. } x \in \partial \Omega \\
\tilde{u}=\gamma_{+} \mu_{s}^{+}-\text {a.e.on } \partial \Omega, \tilde{u}=\gamma_{-} \mu_{s}^{-}-\text {a.e.on } \partial \Omega,
\end{gathered}
$$

iii) We show that $D\left(A_{\delta}\right)$ is dense in $L^{1}(\Omega)$ i.e. $\overline{D\left(A_{\delta}\right)} \|^{\|\cdot\|_{1}}=L^{1}(\Omega)$.

We have $D\left(A_{\delta}\right) \subset L^{\infty}(\Omega) \subset L^{1}(\Omega)$ (since $\Omega$ is bounded). Therefore $\overline{D\left(A_{\delta}\right)^{\|} \|_{1}} \subset L^{1}(\Omega)$. Conversly, let's show that

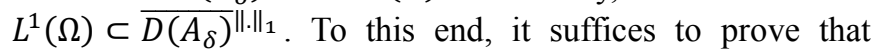
$L^{\infty}(\Omega) \subset \overline{D\left(A_{\delta}\right)} \|^{\|\cdot\|_{1}}$ (since $L^{\infty}(\Omega)$ is dense in $L^{1}(\Omega)$ ). Let $\alpha>0$. Given $v \in \mathfrak{M}_{b}^{p}(\Omega) \cap L^{\infty}(\Omega)$, if we set $u_{\alpha}:=$ $\left(I+\alpha A_{\delta}\right)^{-1} v$, then $\left(u_{\alpha}, \frac{1}{\alpha}\left(v-u_{\alpha}\right)\right) \in A_{\delta}$. So, taking $\phi=0$ as a test function in the definition of the operator $A_{\delta}$, we get

$$
\begin{aligned}
& \int_{\Omega} a\left(u_{\alpha}, \nabla u_{\alpha}\right) \cdot \nabla u_{\alpha} d x+\delta \int_{\partial \Omega} \psi\left(u_{\alpha}\right) u_{\alpha} d \sigma \\
& \leq \frac{1}{\alpha} \int_{\Omega} u_{\alpha} d v-\frac{1}{\alpha} \int_{\Omega}\left|u_{\alpha}\right|^{2} d x-\int_{\partial \Omega} \tilde{u}_{\alpha} d \mu_{\alpha} .
\end{aligned}
$$
that

Using assumption (H2), we deduce from inequality (30)

$$
\begin{gathered}
\lambda_{0}\left\|\nabla u_{\alpha}\right\|_{p}^{p} \leq \frac{1}{\alpha} \int_{\Omega} u_{\alpha} d v-\delta \int_{\partial \Omega} \psi\left(u_{\alpha}\right) u_{\alpha} d \sigma \\
-\int_{\partial \Omega} \tilde{u}_{\alpha} d \mu_{\alpha}-\int_{\Omega} a\left(u_{\alpha}, 0\right) . \nabla u_{\alpha} d x .
\end{gathered}
$$

Using the hypothesis (H3), the monotonicity of $\psi$, properties of $\mu$ and the $L \infty$-estimate on, we get from (31)

$$
\lambda_{0}\left\|\nabla u_{\alpha}\right\|_{p}^{p} \leq \frac{1}{\alpha} C^{\prime}+C
$$

Using the assumption (H3), Hölder inequality and (32), we get

$$
\lim _{\alpha \rightarrow 0} \alpha \int_{\Omega}\left|a\left(u_{\alpha}, \nabla u_{\alpha}\right)\right|=0
$$

On the other hand, if $\phi \in D(\Omega)$, taking $u \alpha+\phi$ and $u \alpha-\phi$ as test functions in the definition of the operator $A_{\delta}$, we get after adding both inequalities

$$
\begin{gathered}
\alpha \int_{\Omega} a\left(u_{\alpha}, \nabla u_{\alpha}\right) \cdot \nabla \phi d x+\alpha \delta \int_{\partial \Omega} \psi\left(u_{\alpha}\right) \phi d \sigma=\int_{\Omega} \phi d v- \\
\int_{\Omega} u_{\alpha} \phi d x-\alpha \int_{\partial \Omega} \tilde{\phi} d \mu_{\alpha}
\end{gathered}
$$

Passing to the limit as $\alpha \rightarrow 0$ in inequality (33), we get

$$
\lim _{\alpha \rightarrow 0} \int_{\Omega} u_{\alpha} \phi d x=\int_{\Omega} \phi d v, \forall \phi \in \mathcal{D}(\Omega)
$$

Since $\left(u_{\alpha}\right)_{\alpha}$ is bounded in $L^{\infty}(\Omega)$ there exists a subsequence $\left(u_{\alpha_{n}}\right)_{n}$ such that $u_{\alpha_{n}} \rightarrow u$ weakly in $L^{p}(\Omega)$. Therefore, using (34), we get $u=v$. As $\left(u_{\alpha}\right)_{\alpha}$ is bounded in
$L^{\infty}(\Omega)$, we have $\|u \alpha\|_{p}^{p} \leq C$. By Lebesgue dominated convergence theorem, $u_{\alpha} \rightarrow v$ in $L^{p}(\Omega)$. As a consequence, $v \in \overline{D\left(A_{\delta}\right)} \|^{\|\cdot\|_{1}}$. This achieves the proof of Theorem 1 .

\section{Entropy Solution}

Before introducing the notion of entropy solutions for the problem (1), we define the following spaces similar to that introduced in $[3,7]$. We note

$$
\mathcal{T}^{1, p}(\Omega):=\left\{\begin{array}{c}
u: \Omega \rightarrow \mathbb{R} \text { measurable; } T_{k}(u) \\
\in W^{1, p}(\Omega) \text { for all } k>0
\end{array}\right\}
$$

Bénilan et al [7] proved that for $u \in \mathcal{T}^{1, p}(\Omega)$ there exists a unique measurable function $w: \Omega \rightarrow \mathbb{R}$ such that $D T_{k}(u)=w \chi_{\{|w|<k\}} \forall k>0 . \quad$ This function $w$ will be denoted by $D u$. Denote by $\mathcal{T}_{t r}^{1, p}(\Omega)$ the subset of $\mathcal{T}^{1, p}(\Omega)$ consisting of the function that can be approximated by functions of $W^{1, p}(\Omega)$ in the following sense: a function $u \in \mathcal{T}^{1, p}(\Omega)$ belongs to $\mathcal{T}_{t r}^{1, p}(\Omega)$ if there exists a sequence $\left(u_{\delta}\right)_{\delta} \in W^{1, p}(\Omega)$ such that:

$$
u_{\delta} \rightarrow u \text { a. e. in } \Omega \text {; }
$$

$D T_{k}\left(u_{\delta}\right) \rightarrow D T_{k}(u)$ weakly in $L^{1}(\Omega)$ for any $k>0$;

There exists a measurable function $v: \partial \Omega \rightarrow \mathbb{R}$ such that $\left(\tau\left(u_{\delta}\right)\right)_{\delta}$ converges a. e. in $\partial \Omega$ to $v$. The function $v$ is called the trace of $u$, denoted $\tau(u)$ or $u$.

Following Sbihi and Wittbold [22], we define an entropy solution of (1) as follows.

Definition 1. For $v \in \mathfrak{M}_{b}^{p}(\Omega)$, a function $u \in \mathcal{T}_{t r}^{1, p}(\Omega)$ is an entropy solution for problem (1) if $u \in L^{1}(\Omega)$ and there exists a measure $\mu \in \mathcal{M}_{b}^{p}(\partial \Omega)$ with

$$
\mu_{r}(x) \in \partial j(x, u(x))+\partial I_{\left[\gamma_{-}(x), \gamma_{+}(x)\right]}(u(x)) \text { a. e. } x \in \partial \Omega
$$

such that for all $\phi \in W^{1, p}(\Omega) \cap L^{\infty}(\Omega)$

$$
\begin{gathered}
\int_{\Omega} a(u, \nabla u) . \nabla T_{k}(u-\phi) d x \leq-\int_{\Omega} u T_{k}(u-\phi) d x+ \\
\int_{\Omega} T_{k}(u-\phi) d v-\int_{\partial \Omega} T_{k}(\tilde{u}-\tilde{\phi}) d \mu, \tilde{u}=\gamma_{+} \mu_{s}^{+}- \\
\text {a.e.on } \partial \Omega, \tilde{u}=\gamma_{-} \mu_{s}^{-}-\text {a.e.on } \partial \Omega .
\end{gathered}
$$

We define an operator $\mathcal{A}$ by the rule:

$$
\in \mathcal{A} \text { if and only if }\left\{\begin{array}{c}
(u, v-u) \\
v \in \mathfrak{M}_{b}^{p}(\Omega) \text { and } \\
u \text { is an entropy solution of problem (1). }
\end{array}\right.
$$

In the following, we use the notation $A_{m, n}$ (resp. $\psi_{m, n}$ ) instead of $A_{\delta}($ resp. $\delta \psi)$, where $\psi_{m, n}(u)=\frac{1}{m} \psi\left(u^{+}\right)-$ $\frac{1}{n} \psi\left(u^{-}\right), m, n \in \mathbb{N}^{*}$.

Theorem 2. The operator $\mathcal{A}$ is $m$-accretive with dense domaine in $L^{1}(\Omega)$ and

$$
\mathcal{A}=\liminf _{m, n \rightarrow+\infty} A_{m, n}
$$


where $\lim \inf _{m, n \rightarrow+\infty} A_{m, n}$ is the operator defined by

$$
\begin{gathered}
(x, y) \in \liminf _{m, n \rightarrow+\infty} A_{m, n} \\
\Leftrightarrow\left\{\begin{array}{c}
\forall m, n>0,\left(x_{m, n}, y_{m, n}\right) \in A_{m, n} \text { and } \\
(x, y)=\liminf _{m, n \rightarrow+\infty}\left(x_{m, n}, y_{m, n}\right) \text { in } X \times X,
\end{array}\right.
\end{gathered}
$$

where $X$ denotes a Banach space.

Proof. We divide the proof into six steps.

Step 1: A priori estimates

Let $v \in \mathfrak{M}_{b}^{p}(\Omega)$. Since $v$ is diffuse, recall that $v=f-$ $\operatorname{div}(F)$ in $\mathcal{D}^{\prime}\left(U_{\Omega}\right)$ with $f \in L^{1}\left(U_{\Omega}\right)$ and $F \in\left(L^{p^{\prime}}\left(U_{\Omega}\right)\right)^{N}$ where $\left(U_{\Omega}\right)$ is the open bounded subset of $\mathbb{R}^{N}$ which extend $\Omega$ via the operator $E$. We approximate $f$ by $f_{m, n}=(f \wedge m) \vee(-n) \in L^{\infty}(\Omega)$ nondecreasing in $m$, nonincreasing in $n$. Note that $\left\|f_{m, n}\right\|_{1} \leq\|f\|_{1}$. Let $\left(F_{m, n}\right)_{m, n \geq 1} \subset C_{0}^{\infty}\left(U_{\Omega}\right)$ be a sequence such that $F_{m, n} \rightarrow F$ strongly in $\left(L^{p^{\prime}}\left(U_{\Omega}\right)\right)^{N}$, as $m, n \rightarrow+\infty$. For any $m, n \geq 1$ we set $\tilde{F}_{m, n}=\chi_{\Omega} F_{m, n}$ and $v_{m, n}=f_{m, n}-\operatorname{div}\left(\tilde{F}_{m, n}\right)$. For any $m, n \geq 1$ one has $v_{m, n} \in \mathfrak{M}_{b}^{p}(\Omega), v_{m, n}-v$ in $\mathcal{M}_{b}\left(U_{\Omega}\right)$ and $v_{m, n} \in L^{\infty}(\Omega)$ Furthermore, for any $k>0$ and any $\xi \in \mathcal{T}^{1, p}(\Omega)$

$$
\left|\int_{\Omega} T_{k}(\xi) d v_{m, n}\right| \leq k C(\nu, \Omega)
$$

By Theorem 1, $v_{m, n} \in R\left(I+A_{m, n}\right)$ and there exists $u_{m, n} \in W^{1, p}(\Omega) \cap L^{\infty}(\Omega)$ and a measure $\mu_{m, n} \in \mathcal{M}_{b}^{p}(\partial \Omega)$ satisfying

$$
\begin{gathered}
\left(\mu_{m, n}\right)_{r}(x) \in \partial j\left(x, u_{m, n}(x)\right) \\
+\partial I_{\left[\gamma_{-}(x), \gamma_{+}(x)\right]}\left(u_{m, n}(x)\right) \text { a. e. } x \in \partial \Omega,
\end{gathered}
$$

such that for all $\phi \in W^{1, p}(\Omega) \cap L^{\infty}(\Omega)$,

$$
\begin{gathered}
\int_{\Omega} a\left(u_{m, n}, \nabla u_{m, n}\right) \cdot \nabla\left(u_{m, n}-\phi\right) d x+\int_{\partial \Omega} \psi_{m, n}\left(u_{m, n}\right)\left(u_{m, n}-\phi\right) d \sigma \\
\leq \int_{\Omega}\left(u_{m, n}-\phi\right) d v_{m, n}-\int_{\Omega} u_{m, n}\left(u_{m, n}-\phi\right) d x-\int_{\partial \Omega}\left(\tilde{u}_{m, n}-\tilde{\phi}\right) d \mu_{m, n} \\
\text { and } \tilde{u}_{m, n}^{ \pm}=\gamma_{ \pm}\left(\mu_{m, n}\right)_{s}^{ \pm} \text {a. e. on } \partial \Omega .
\end{gathered}
$$

In the following, let $k>0$ be fixed. Using $\phi=u_{m, n}-T_{k}\left(u_{m, n}\right)$ as a test function in (37) and applying hypothesis $\left(H_{2}\right)$, we obtain

$$
\begin{gathered}
\lambda_{0} \int_{\Omega}\left|\nabla T_{k}\left(u_{m, n}\right)\right|^{p} d x+\frac{1}{m} \int_{\partial \Omega} T_{k}\left(u_{m, n}\right) \psi\left(u_{m, n}^{+}\right) d \sigma-\frac{1}{n} \int_{\partial \Omega} T_{k}\left(u_{m, n}\right) \psi\left(u_{m, n}^{-}\right) d \sigma \\
\leq \int_{\Omega} T_{k}\left(u_{m, n}\right) d v_{m, n}-\int_{\Omega} T_{k}\left(u_{m, n}\right) u_{m, n} d x \\
-\int_{\partial \Omega} T_{k}\left(\tilde{u}_{m, n}\right) d \mu_{m, n}-\int_{\Omega} a\left(u_{m, n}, 0\right) \cdot \nabla T_{k}\left(u_{m, n}\right) d x
\end{gathered}
$$

By Gauss-Green Formula and hypothesis $\left(H_{3}\right)$, we have

$$
\left|\int_{\Omega} a\left(u_{m, n}, 0\right) \cdot \nabla T_{k}\left(u_{m, n}\right) d x\right| \leq C
$$

where $C$ is a constant depending on $k$. Then, from inequality (38), according to the monotonicity of $\psi$, we conclude

$$
\lambda_{0} \int_{\Omega}\left|\nabla T_{k}\left(u_{m, n}\right)\right|^{p} d x \leq C
$$

Thus $\left(T_{k}\left(u_{m, n}\right)\right)_{m, n}$ is a bounded subset of $W^{1, p}(\Omega)$. Hence, after passing to a suitable subsequence if necessary, $\left(T_{k}\left(u_{m, n}\right)\right)_{m, n}$ is weakly convergent in $W^{1, p}(\Omega)$. Then, $T_{k}\left(u_{m, n}\right) \rightarrow v_{k}$ in $L^{p}(\Omega)$ as $m, n \rightarrow \infty$. We may also assume that $T_{k}\left(u_{m, n}\right) \rightarrow g_{k}$ in $\left(L^{p}(\Omega)\right)^{N}$ as $m, n \rightarrow \infty$.

Now, we must prove the convergence almost everywhere of $u_{m, n}$. As $A_{m, n}$ is $T$-accretive in $L^{1}(\Omega)$, we have for all $m \geq m^{\prime}$,

$$
\int_{\Omega}\left(u_{m^{\prime}, n}-u_{m, n}\right)^{+} d x \leq \int_{\Omega}\left(f_{m^{\prime}, n}-f_{m, n}\right)^{+} d x .
$$

As $\left(f_{m, n}\right)$ is nondecreasing in $m$ and nonincreasing in $n$, we deduce that $\left(u_{m, n}\right)_{m}$ is nondecreasing and $\left(u_{m, n}\right)_{n}$ is nonincreasing. Since $\left(u_{m, n}\right)_{m, n}$ is uniformly bounded then we deduce that

$$
u_{m, n} \uparrow u_{n} \text { when } m \rightarrow+\infty \text { and } u_{n} \downarrow u \text { as } n \rightarrow+\infty \text {. }
$$

By applying Lebesgue dominated convergence theorem, we get

$$
u_{m, n} \uparrow_{m} u^{n} \downarrow_{n} u, u_{m, n} \downarrow_{n} u_{m} \uparrow_{m} u \text { in } L^{1}(\Omega)
$$

Therefore, from (41) we get the convergence of $\left(u_{m, n}\right)_{m, n}$ to $u$ in $L^{1}(\Omega)$ and also the convergence almost everywhere on $\Omega$. Then, we conclude that $v k=T k(u)$ and $=$ $\nabla T k(u)$. Therefore, $T k(u) \in W 1, p(\Omega)$ for all $k>0$. Consequently, $u \in \mathcal{T}^{1, p}(\Omega)$.

Finally, we show exactly as Andreu et al [3], that ( $\tau$ (um, n)) $\mathrm{m}$, n converge a. e. on $\partial \Omega$ and then $u \in \mathcal{T}_{t r}^{1, p}(\Omega)$.

Step 2: Existence of the measure $\mu$

It remains to show the existence of a measure $\mu \in$ $\mathcal{M}_{b}^{p}(\partial \Omega)$ such that $\mu_{m, n} \rightarrow \mu$ strongly in $\mathcal{M}_{b}^{p}(\partial \Omega)$. Let $u_{m, n}^{\lambda}$ be a solution of the problem 


$$
\begin{gathered}
\int_{\Omega} a\left(u_{m, n}^{\lambda}, \nabla u_{m, n}^{\lambda}\right) \cdot \nabla \varphi d x+\frac{1}{m} \int_{\partial \Omega} \psi\left(u_{m, n}^{\lambda,+}\right) \varphi d \sigma-\frac{1}{n} \int_{\partial \Omega} \psi\left(u_{m, n}^{\lambda,-}\right) \varphi d \sigma \\
=\int_{\Omega} \varphi d v_{m, n}-\int_{\Omega} u_{m, n}^{\lambda} \varphi d x-\int_{\partial \Omega} \beta_{\lambda}\left(., u_{m, n}^{\lambda}\right) \varphi d \sigma .
\end{gathered}
$$

for all $\varphi \in W^{1, p}(\Omega) \cap L^{\infty}(\Omega)$.

We know from Theorem 1 that $\left\|\beta_{\lambda}\left(., u_{m, n}^{\lambda}\right)\right\|_{1}$ is uniformly bounded by a constant $C$ independent of $\lambda$, thus $\beta_{\lambda}\left(., u_{m, n}^{\lambda}\right) \rightarrow \mu_{m, n}$ in $\mathcal{M}_{b}(\partial \Omega)$ as $\lambda \rightarrow 0$. Therefore

$$
\left\|\mu_{m, n}\right\|_{\mathcal{M}_{b}(\partial \Omega)} \leq \liminf _{\lambda \rightarrow 0}\left\|\beta_{\lambda}\left(., u_{m, n}^{\lambda}\right)\right\|_{\mathcal{M}_{b}(\partial \Omega)} \leq C
$$

and we deduce, after extracting a subsequence if necessary that $\mu_{m, n} \rightarrow \mu$ weakly in $\mathcal{M}_{b}(\partial \Omega)$ as $m, n \rightarrow \infty$. In order to prove the strong convergence of $\mu_{m, n}$, we use the following comparison result.

Lemma 3. (see [22], Lemma 4.1) Assume that $\widetilde{m} \geq$ $m, \tilde{n} \geq n$ and $v_{m, n}, v_{\widetilde{m}, n} \in L^{\infty}(\Omega)$. Let $u_{m, n}^{\lambda}, u_{\tilde{m}, n}^{\lambda}$ be the weak solutions which satisfy (42). Then

$$
u_{m, \tilde{n}}^{\lambda} \leq u_{m, n}^{\lambda} \leq u_{\tilde{m}, n}^{\lambda} \text { a.e. in } \Omega
$$

and

$$
\begin{gathered}
\beta_{\lambda}\left(., u_{m, \tilde{n}}^{\lambda}\right) \leq \beta_{\lambda}\left(., u_{m, n}^{\lambda}\right) \leq \beta_{\lambda}\left(., u_{\tilde{m}, n}^{\lambda}\right) \text { a. e. in } \partial \Omega \\
\begin{array}{r}
\int_{\Omega} a\left(u_{m, n}, \nabla u_{m, n}\right) . \nabla \varphi d x+\frac{1}{m} \int_{\partial \Omega} \psi\left(u_{m, n}^{+}\right) \varphi d \sigma-\frac{1}{n} \int_{\partial \Omega} \psi\left(u_{m, n}^{-}\right) \varphi d \sigma \\
\quad=\int_{\Omega} \varphi d v_{m, n}-\int_{\Omega} u_{m, n} \varphi d x-\int_{\partial \Omega} \beta_{\lambda}\left(., u_{m, n}\right) \varphi d \sigma .
\end{array}
\end{gathered}
$$

Since $\left(T_{k}\left(u_{m, n}\right)\right)_{m, n}$ is bounded in $W^{1, p}(\Omega)$ then, thanks to the growth assumption $\left(H_{3}\right)$, there exists a vector fields $\chi_{k} \in\left(L^{p^{\prime}}(\Omega)\right)^{N}$ such that $a\left(T_{k}\left(u_{m, n}\right), \nabla T_{k}\left(u_{m, n}\right)\right) \rightarrow \chi_{k}$ weakly in $\left(L^{p^{\prime}}(\Omega)\right)^{N}$ as $m, n \rightarrow+\infty$, for all $k \in \mathbb{N}^{*}$. We prove as Sbihi and Wittbold ([22], Theorem 4.1), via a pseudo-monotonicity argument, that

$$
\operatorname{div} \chi_{k}=\operatorname{div} a\left(T_{k}(u), \nabla T_{k}(u)\right) \text { in } \mathcal{D}^{\prime}(\Omega)
$$

Step 4: Passage to the limit in Equation (43).

Taking $\varphi=S\left(u_{m, n}-\phi\right)$ as a test function in (42), where $S \in \mathcal{P}=$ $\left\{p \in C^{1}(\mathbb{R}) ; p(0)=0,0 \leq p^{\prime} \leq\right.$ $1, \operatorname{supp}\left(p^{\prime}\right)$ is compact $\}, \phi \in W^{1, p}(\Omega) \cap L^{\infty}(\Omega)$ and define

$$
\begin{gathered}
\int_{\Omega} S\left(u_{m, n}-\phi\right) d v_{m, n}=\int_{\Omega} E\left(S\left(u_{m, n}-\phi\right)\right) d v_{m, n} \\
=\left\langle v_{m, n}, E\left(S\left(u_{m, n}-\phi\right)\right)\right\rangle \\
=\int_{\Omega} f_{m, n} S\left(u_{m, n}-\phi\right) d x+\int_{U_{\Omega}} F_{m, n} . \nabla E\left(\chi_{\Omega} S\left(u_{m, n}-\phi\right)\right) d x
\end{gathered}
$$

By the Lebesgue dominated convergence theorem, we have for the first term of the right hand side of (46),

$$
\lim _{m, n \rightarrow \infty} \int_{\Omega} f_{m, n} S\left(u_{m, n}-\phi\right) d x=\int_{\Omega} f S(u-\phi) d x
$$

As $\left|\chi_{\Omega} S\left(u_{m, n}-\phi\right)\right| \leq C$ for all $m, n \geq 1$, then we use the inequality

$$
\|E(v)\|_{W_{0}^{1, p}\left(U_{\Omega}\right)} \leq c\|v\|_{W^{1, p}(\Omega)}, \forall v \in W^{1, p}(\Omega)
$$

to conclude that the sequence $\left(E\left(S\left(u_{m, n}-\phi\right)\right)\right)_{m, n \geq 1}$ is uniformly bounded in $W_{0}^{1, p}\left(U_{\Omega}\right)$. We also have $E\left(\chi_{\Omega} S\left(u_{m, n}-\phi\right)\right) \rightarrow S(u-\phi)$ a. e. in $U_{\Omega}$ as $m, n \rightarrow$ $+\infty$. Then, $\nabla E\left(\chi_{\Omega} S\left(u_{m, n}-\phi\right)\right) \rightarrow \nabla S(u-\phi) \quad$ in $\left(L^{p}\left(U_{\Omega}\right)\right)^{N}$.

Finally, we get for the second term in the right hand side of (46), 


$$
\lim _{m, n \rightarrow \infty} \int_{U_{\Omega}} F_{m, n} \cdot \nabla E\left(\chi_{\Omega} S\left(u_{m, n}-\phi\right)\right) d x=\int_{U_{\Omega}} F . \nabla E\left(\chi_{\Omega} S(u-\phi)\right) d x
$$

Using (47) and (48), we get from (46),

$$
\begin{aligned}
\lim _{m, n \rightarrow \infty} \int_{\Omega} S\left(u_{m, n}-\phi\right) d v_{m, n} & =\int_{\Omega} f S(u-\phi) d x+\int_{U_{\Omega}} F \cdot \nabla E\left(\chi_{\Omega} S(u-\phi)\right) d x \\
& =\int_{U_{\Omega}} f E\left(\chi_{\Omega} S(u-\phi)\right) d x+\int_{U_{\Omega}} F \cdot \nabla E\left(\chi_{\Omega} S(u-\phi)\right) d x \\
& =\quad\left\langle v, E\left(\chi_{\Omega} S(u-\phi)\right)\right\rangle \\
& =\int_{U_{\Omega}} E\left(\chi_{\Omega} S(u-\phi)\right) d v \\
& =\int_{\Omega} S(u-\phi) d v
\end{aligned}
$$

We also note that

$$
\begin{gathered}
\int_{\partial \Omega} \psi_{m, n}\left(u_{m, n}\right) S\left(u_{m, n}-\phi\right) d \sigma \\
=\int_{\partial \Omega}\left[\psi_{m, n}\left(u_{m, n}\right)-\psi_{m, n}(\phi)\right] S\left(u_{m, n}-\phi\right) d \sigma+\int_{\partial \Omega} \psi_{m, n}(\phi) S\left(u_{m, n}-\phi\right) d \sigma \\
=\int_{\partial \Omega}\left[\psi_{m, n}\left(u_{m, n}\right)-\psi_{m, n}(\phi)\right] S\left(u_{m, n}-\phi\right) d \sigma+\frac{1}{m} \int_{\partial \Omega} \psi\left(\phi^{+}\right) S\left(u_{m, n}-\phi\right) d \sigma \\
-\frac{1}{n} \int_{\partial \Omega} \psi\left(\phi^{-}\right) S\left(u_{m, n}-\phi\right) d \sigma .
\end{gathered}
$$

As the functions $\psi m, n$ and $S$ are nondecreasing, we get

$$
\int_{\partial \Omega}\left[\psi_{m, n}\left(u_{m, n}\right)-\psi_{m, n}(\phi)\right] S\left(u_{m, n}-\phi\right) d \sigma \geq 0
$$

On the other hand, as $\psi, u m, n$ and $S$ are bounded, then

$$
\begin{gathered}
\lim _{m, n \rightarrow \infty} \frac{1}{m} \int_{\partial \Omega} \psi\left(\phi^{+}\right) S\left(u_{m, n}-\phi\right) d \sigma \\
=0 \text { and } \lim _{m, n \rightarrow \infty} \frac{1}{n} \int_{\partial \Omega} \psi\left(\phi^{-}\right) S\left(u_{m, n}-\phi\right) d \sigma=0 .
\end{gathered}
$$

Therefore,

$$
\lim _{m, n \rightarrow \infty} \int_{\partial \Omega} \psi_{m, n}\left(u_{m, n}\right) S\left(u_{m, n}-\phi\right) d \sigma \geq 0
$$

To complete the proof, it remains to show that $\mu$ verifies $\mu_{r} \in \partial j(., u)+\partial I_{\left[\gamma_{-} \gamma_{+}\right]}(u)$ a.e. $\partial \Omega, \tilde{u}=\gamma_{+} \mu_{s}^{+}$a. e. on $\partial \Omega, \tilde{u}=\gamma_{-} \mu_{s}^{-}$a. e. on $\partial \Omega$ and

$$
\lim _{m, n \rightarrow \infty} \int_{\partial \Omega} S\left(\tilde{u}_{m, n}-\tilde{\phi}\right) d \mu_{m, n}=\int_{\partial \Omega} S(\tilde{u}-\tilde{\phi}) d \mu
$$

We know from the proof of Theorem 1 (part ii)) that $\mu_{m, n} \in \partial \mathcal{J}\left(u_{m, n}\right)$, thus

$$
\begin{gathered}
\left(\mu_{m, n}\right)_{r}(x) \in \partial j\left(x, u_{m, n}(x)\right) \\
+\partial I_{\left[\gamma_{-}(x), \gamma_{+}(x)\right]}\left(u_{m, n}(x)\right) \text { a. e. } x \in \partial \Omega .
\end{gathered}
$$

As $\quad u_{m, n} \rightarrow u$ a. e. on $\partial \Omega$ and $\left\|\left(\mu_{m, n}\right)_{r}-\mu_{r}\right\|_{L^{1}(\partial \Omega)} \leq$ $\left\|\mu_{m, n}-\mu\right\|_{\mathcal{M}_{b}(\partial \Omega)} \rightarrow 0$ as $m, n \rightarrow+\infty$, then $\mu_{r}(x) \in \partial j(x, u(x))+\partial I_{\left[\gamma_{-}(x), \gamma_{+}(x)\right]}(u(x))$ a.e. $x \in \partial \Omega$.
On the other hand, we have

$$
\tilde{u}_{m, n}=\gamma_{+}\left(\mu_{m, n}\right)_{s}^{+} \text {a. e. on } \partial \Omega, \tilde{u}_{m, n}=
$$
$\gamma_{-}\left(\mu_{m, n}\right)_{s}^{-}$a. e. on $\partial \Omega$, which are equivalent to

$$
\begin{gathered}
\int_{\partial \Omega}\left(\gamma_{+}-\tilde{u}_{m, n}\right) d\left(\mu_{m, n}\right)_{s}^{+}=0 \\
\text { and } \int_{\partial \Omega}\left(\gamma_{-}-\tilde{u}_{m, n}\right) d\left(\mu_{m, n}\right)_{s}^{-}=0
\end{gathered}
$$

As $u$ is bounded on $\partial \Omega$ and $\left(\mu_{m, n}\right)_{s} \rightarrow \mu_{s}$ strongly on $\mathcal{M}_{b}(\partial \Omega)$ as $m, n \rightarrow+\infty$ then, by passing to the limit in the last both integrals according to Lebesgue dominated convergence theorem, we obtain

$$
\int_{\partial \Omega}\left(\gamma_{+}-\tilde{u}\right) d \mu_{s}^{+}=0 \text { and } \int_{\partial \Omega}\left(\gamma_{-}-\tilde{u}\right) d \mu_{s}^{-}=0,
$$

which are equivalent to $\tilde{u}=\gamma_{ \pm} \mu_{s}^{ \pm}$a.e. on $\partial \Omega$.

As $u_{m, n} \rightarrow u$ a.e. on $\partial \Omega$ and $\mu_{m, n} \rightarrow \mu$ weakly in $\mathcal{M}_{b}(\partial \Omega)$ then, using Lebesgue dominated convergence theorem, we get (51).

Finally, collecting together all the limits (44)-(45) and (49)-(51), we conclude that:

$$
\begin{gathered}
\int_{\Omega} a(u, \nabla u) \cdot \nabla S(u-\phi) d x+\int_{\partial \Omega} S(\tilde{u}-\tilde{\phi}) d u \\
\leq \int_{\Omega} S(u-\phi) d v-\int_{\Omega} u S(u-\phi) d x
\end{gathered}
$$

for all $\phi \in W^{1, p}(\Omega) \cap L^{\infty}(\Omega)$. 
Taking $S$ as an approximation of $T_{k}$, we get the desired entropy inequality.Therefore, we have shown that, for all $v \in \mathfrak{M}_{b}^{p}(\Omega) \cap L^{\infty}(\Omega),\left(I+A_{m, n}\right)^{-1} v$ converges in $L^{1}(\Omega)$ to an entropy solution of the problem (1), hence

$$
\liminf _{m, n \rightarrow \infty} A_{m, n} \subset \mathcal{A} .
$$

For the inverse inclusion we refer to the step below.

Step 5: The accretivity of $\mathcal{A}$

To prove the accretivity of $\mathcal{A}$, we show as Sbihi and Wittbold ([22], Theorem 4.1) and as in Theorem 1 of section 3,

$$
\int_{\Omega}|w-v| d x \leq \int_{\Omega}|f-g| d x,
$$

where $f, g \in L^{1}(\Omega)$ provide from the decomposition of the measures $\quad v_{1}=f-\operatorname{div}\left(F_{1}\right) \in w+\mathcal{A} w \quad$ and $\quad v_{2}=g-$ $\operatorname{div}\left(F_{2}\right) \in v+\mathcal{A v}$.

Step 6: $D(A)$ is dense in $L 1(\Omega)$

For this, we show that $L^{\infty}(\Omega) \subset \overline{D(\mathcal{A})}\left\|^{\|}\right\|_{1}$. Let $u \in$ $L^{\infty}(\Omega)$. Consider $u_{m, n}^{\alpha}$ and $u_{\alpha}, \alpha>0$ such that

$$
u_{m, n}^{\alpha}+\alpha A_{m, n} u_{m, n}^{\alpha} \ni u \text { and } u_{\alpha}+\alpha \mathcal{A} u_{\alpha} \ni u
$$

We know from Theorem 1 that $D\left(A_{m, n}\right)$ is dense in $L^{1}(\Omega)$, for all $m, n \in \mathbb{N}^{*}$, we have

$$
u_{m, n}^{\alpha} \rightarrow u \operatorname{in} L^{1}(\Omega) \text { as } \alpha \rightarrow 0 .
$$

As Sbihi and Wittbold ([22], Theorem 4.1), we show that

$$
u_{m, n}^{\alpha} \rightarrow u \text { in } L^{1}(\Omega) \text { as } m, n \rightarrow+\infty .
$$

Then, we deduce $u \in \overline{D(\mathcal{A})^{\| .} \|_{1}}$.

Corollary 1. Under the assumptions of Theorem 2, we have the existence and uniqueness of entropy solution $u$ for the problem (1).

\section{Conclusion}

In this paper we proved existence and uniqueness of entropy solution for nonlinear elliptic problem with nonlinear boundary conditions of type graph and measure data. The main difficulty is that, when one uses the integration by parts formula in the variational approach, it appear at the boundary, for the part of the measure-data $v$ which is in $W^{1, p}(\Omega)$, a term which cannot vanish. In order to treat this difficulty, we consider a smooth domain $\Omega$ in order to work with the space $W_{0}^{1, p}(\Omega)$ and to going back later to the space $W^{1, p}(\Omega)$. For uniqueness, we prove a comparison principle for entropy solutions by using the approximation method. Not that assumption $\left(H_{4}\right)$, used to prove uniqueness of entropy solution is not optimal. In fact, it is sufficient to assume that $a$ satisfies some Hölder type continuity and a certain growth restriction in $r$ instead (see [4]). In a forthcoming paper, we will study the same problem with $u$ replaced by $b(u)$ and with the same nonlinear boundary conditions and measure data. The case where $b$ is continuous nondecreasing corresponds to the stationary problem associated with the elliptic-parabolic evolution problem arising as a model of fluid flow through porous media. In this type of problems, from the view point of applications, it is essential to study general nonlinear boundary conditions. The more general case where $b$ is a multivalued monotone graph corresponds to a Stefan problem arising in applications in presence of phase transitions.

\section{References}

[1] H. W. Alt, S. Luckhaus; Quasilinear Elliptic-Parabolic Differential Equations, Math. Z. 183 (1983), 311-341.

[2] K. Ammar, Solutions entropiques et renormalisées de quelques E. D. P non linaires dans L1, Thesis, Strasbourg, 2003.

[3] F. Andreu, J. M. Mazon, S. Segura de L'eon, J. Toledo, Quasi-linear elliptic and parabolic equations in L1 with nonlinear boundary conditions, Adv. Math. Sci. Appl. 7, No. 1 (1997), 183-213.

[4] B. Andreianov, F. Bouhssis, Uniqueness for an elliptic-parabolic problem with Neumann boundary condition, J. Evol. Equ. 4 (2004), no. 2, 273-295.

[5] V. Barbu, Nonlinear semigroups and Differential Equations in Banach Spaces, Noordhoff, Leyden, 1976.

[6] $\mathrm{Ph}$. B'enilan, Equations d'evolution dans un espace de Banach quelconque et applications, Thesis, Orsay, 1972.

[7] Ph. B'enilan, L. Boccardo, T. Gallou"et, R. Gariepy, M. Pierre, J. L. Vasquez, An L1 -theory of existence and uniqueness of solutions of nonlinear elliptic equations, Ann. Scuola Norm. Sup. Pisa 22 (1995), 241-273.

[8] Ph. B'enilan, H. Br'ezis, M. G. Crandall, A semilinear equation in L1, Ann. Scuola Norm. Sup. Pisa 2 (1975), 523-555.

[9] Ph. B'enilan, J. Carrillo, P. Wittbold, Renormalized and entropy solutions of scalar conservation laws, Ann. Scuola Norm. Sup. Pisa 29 (2000), 313-327.

[10] Ph. B'enilan, B. G. Crandall, A. Pazy, Evolutions Equations Governed by accretive Operators, Forth- coming book.

[11] Ph. B'enilan, P. Wittbold, on mild and weak solutions of elliptic-parabolic equations, Adv. Diff. Equ. 1 (1996), 1053-1073.

[12] L. Boccardo, T. Gallou"et, L. Orsina, Existence and uniqueness of entropy solutions for nonlinear elliptic equations with measure data, Ann. Inst. Henri Poincar'e, 13, 5 (1196), 539-551.

[13] G. Bouchitt'e, Calcul des variations en cadre non r'eflexif. Repr'esentation et relaxation de fonction-nelles int'egrales sur un espace de mesures. Applications en plasticit'e et homog'enisation. Th'ese de Doctorat d'Etat. Perpignan, 1987.

[14] G. Bouchitt'e, Conjugu'e et sous-diff'erentiel d'une fonctionnelle int' egrale sur un espace de Sobolev, C. R. Acad. Sci. Paris S'er. I Math. 307 (1988), no., 79-82.

[15] H. Br'ezis; Analyse Fonctionnelle: Th'eorie et Applications, Paris, Masson (1983).

[16] J. Carrillo, Entropy solutions for nonlinear degenerate problems, Arch. Ration. Mech. Anal, 147 (1999), 269-361. 
[17] R. Diperna, P. L. Lions, On the Cauchy problem for the Boltzman equation: global existence and stability, Ann. of Math. 130 (1989), 321-366.

[18] N. Dunfort, L. schwartz, Linear Operators, part I, Pure and Applied Mathematics, Vol VII.

[19] C. B. Jr. Morrey, Multiple Integrals in the Calculus of Variations, Springer-Verlag, 1966.

[20] J. Ne cas, Les M'ethodes Directes en Th'eorie des Equations Elliptiques, Masson et Cie, Paris, 1967.

[21] A. Prignet, Conditions aux limites non homog'enes pour des probl'emes elliptiques avec second membre Mesure, Ann. Fac. Sci. Toulouse, 5 (1997), 297-318.
[22] K. Sbihi \& P. Wittbold, Entropy solution of a quasilinear elliptic problem with nonlinear boundary condition, Commun. Appl. Anal. 11 (2007), no. 2, 299-325.

[23] R. E. Showalter, Monotone Operators in Banach Space and Nonlinear Partial Differential Equations, American Mathematical Society, Mathematical Surveys and Monographs, Vol 49, ISSN, 0076-5376, 1997.

[24] F. Simondon, Etude de l'equation $\partial \mathrm{t} \mathrm{b}(\mathrm{u})-\operatorname{div} \mathrm{a}(\mathrm{b}(\mathrm{u}), \mathrm{Du})$ $=0$ par la m'ethode des semi-groupes dans L1 $(\Omega)$, Publ. Math. Besanon, Analyse non lin'eaire 7 (1983). 